\title{
Application of CRISPR/Cas9 genome editing system for molecular breeding of orchids
}

\author{
Endang Semiarti ${ }^{1,}{ }^{*}$, Sri Nopitasari ${ }^{1}$, Yuli Setiawati ${ }^{1}$, Muhammad Dylan Lawrie ${ }^{1}$, Aziz Purwantoro ${ }^{2}$, Jaka Widada ${ }^{2}$, Kana \\ Ninomiya $^{3}$, Yuuki Asano ${ }^{4}$, Shogo Matsumoto $^{3}$, Yasushi Yoshioka ${ }^{4}$ \\ ${ }^{1}$ Faculty of Biology, Universitas Gadjah Mada, Indonesia \\ ${ }^{2}$ Faculty of Agriculture, Universitas Gadjah Mada, Indonesia \\ ${ }^{3}$ Graduate School of Bioagricultural Sciences, Nagoya University, Japan \\ ${ }^{4}$ Division of Biological Science, Graduate School of Science, Nagoya University, Japan \\ *Corresponding author: endsemi@ugm.ac.id
}

SUBMITTED 24 January 2020 REVISED 17 April 2020 ACCEPTED 27 May 2020

\begin{abstract}
Orchid is an important ornamental plant in Indonesia due to their natural beauty of flowers. In the tropical forest, orchids are being acquired for trading and commercial market. Thus, the effort is required to proliferate orchid in large quantities for conservation and improve the floral variation for plant breeding. The purpose of this study is to develop a firmed methodology of molecular breeding of orchids using CRISPR/Cas9 KO system. The plant material used was Phalaenopsis amabilis protocorms growth on NP medium+pepton ( $2 \mathrm{~g} / \mathrm{L})$. Protocorm were submerged in the culture of Agrobacterium tumefaciens that Ti-plasmid had been filled with a T-DNA construct of a pRGEB32 vector harboring sgRNA with PDS3 sequence. Detection for transformants was confirmed by PCR using HPT primers (545 bp), Cas9 primers (402 bp), PDS primers (280 bp) and trnL-F (1200 bp) as an internal control. The results showed that 0.96\% PDS transformants were obtained from PDS3T2 lines. Several transformant showed pale leaf color compared to non-transformant plants. This study suggests that the target gene has successfully edited by CRISPR/Cas9 system and could be applied for that functional gene editing in orchids.
\end{abstract}

KEYWORDS CRISPR/Cas9; Genome editing; Molecular breeding; Orchid; Phytoene desaturase (PDS)

\section{Introduction}

Orchid (Familia: Orchidaceae) is an important horticultural plant in Indonesia. Some orchids, such as Dendrobium and Phalaenopsis, are known as popular potted ornamental plants for their beautiful and long-lasting flowers (Tong et al. 2019; Cai et al. 2015). On the contrary, the shooting emergences of orchids in term of seedling production are difficult to achieve naturally and the flowering time is very time consuming. These conditions are common problems in orchid breeding. Therefore it is necessary to overcome those limitations in order to promote and accelerate the seedling production and flowering time. Those problems could be solved through CRISPR/Cas9 genome editing system to edit shooting and flowering genes. CRISPR/Cas9 is the latest genome editing technology that has been already used in various animals and plants including model and non-model ones. With its high efficiency, CRISPR/Cas9 could be used for targeting biallelic genes simultaneously (Tsutsui and Higashiyama 2016).

Conventional genetic engineering strategy has several issues and limitations, one of which is the complexity associated with the manipulation of large genomes of higher plants. However, creation of novel tools for breeding and biotechnology, an application area of genetic engineering, has received significant focus resulting in accelerated development of useful tools. At the present, gene editing techniques have the potential to substantially accelerate plant breeding.

Genome editing is a group of technologies that give scientists the ability to change an organism's DNA (Hsu et al. 2014). Targeted genome editing using artificial nucleases has the potential to accelerate basic research as well as plant breeding by providing the means to modify genomes rapidly in a precise and predictable manner. The Clustered Regularly Interspaced Short Palindromic Repeat (CRISPR)/CRISPR-associated protein 9 (Cas9) system is a new developed tool for the introduction of site-specific double-stranded DNA breaks (Liu et al. 2017). Cas9 protein induces double-strand breaks (DBS) (Shen et al. 2017), which then repaired by the intracellular system. These technologies allow genetic material to be added, removed, or altered at particular locations in the genome, 
it can be edits the form of point mutation, insertion, and deletion through the non-homologous end-joining (NHEJ) pathway (Chiruvella et al. 2013) or by gene replacement through homology-directed recombination (HDR) (Hahn et al. 2018).

This research objective is to develop CRISPR/Cas9 method for use in orchid in order to produce desirable allele in less than 20 months compared to the conventional breeding that need several years. In this preliminary study, we used Phytoene desaturase (PDS3) gene which is a ratelimiting enzyme in carotenoid synthesis. The PDS3 gene converts phytoene to colored $\xi$-carotene in a two-step desaturation reaction (Wang et al. 2009). PDS gene encoding the phytoene desaturase enzyme is involved in carotenoid biosynthesis pathway (Naing et al. 2019), therefore mutations in those genes cause albino phenotype (Koschmieder et al. 2017). In general PDS3 gene is widely used as a common marker gene in many plant species due to its ease of detection. Based on the easy detection of mutation phenotypically, this gene was chosen as target for genome editing. This is a preliminary study to find out how high mutation frequencies can be obtained with the CRISPR/Cas9 genome editing system in $P$. amabilis orchids, which can be further applied to edit other functional genes in orchids.

\section{Materials and Methods}

\subsection{Plant Materials}

Mature $P$. amabilis capsules were sterilized with $75 \%$ ethyl alcohol, $0.1 \%(\mathrm{w} / \mathrm{v})$ natrium hydrochloride, then washed with sterile distilled water. After sterilization, the capsule was opened by cutting it in half longitudinally, then the seeds were sown on NP + peptone media (2 g/L). Cultures were maintained in an incubator with a temperature of day/night $\pm 25^{\circ} \mathrm{C}$, orchid seeds then grew into a protocorm (developing orchid embryo). In general, protocorms could be used as targets because of their high regeneration capacity.

\subsection{Vector Construction and Targets Selection}

The vector pRGEB32 in Agrobacterium strain EHA105 was used to transfer the T-DNA which contain CRISPR/Cas9 into orchid plant (Figure 1). The T-DNA

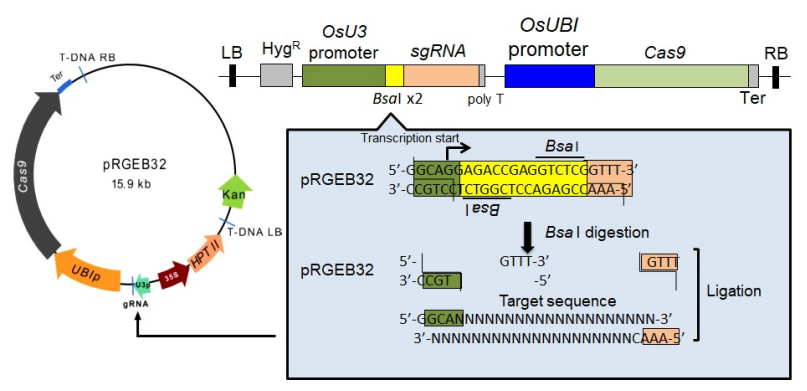

FIGURE 1 Plasmid pRGEB32 construction containing T-DNA carried CRISPR/Cas9 (Xie et al. 2015). region of this vector harbors hygromycin phosphotransferase $\left(\mathrm{Hyg}^{\mathrm{R}}\right)$ as the selection marker genes. $\mathrm{Hyg}^{R}$ gene was driven by the CaMV 35S (35S) promoter and ended by the 35S' terminator, and cloned into the T-DNA in the pRGEB32 vector. Agrobacterium-mediated transformation was conducted according the protocol described by Semiarti et al. (2010, 2011) for genetic transformation into plant.

To create a genome edited $P$. amabilis by using CRISPR/Cas9 system, an RNA-guided genome editing vector line pRGEB32 (Bsa I)-Cas9 was used for expressing engineered sgRNA and Cas9 in cells. The sgRNA expression cassette was driven by the U3 promoter. This promoter was a small nuclear RNA (snRNA) promoter that initiates transcription at an adenine nucleotide and drives the high expression of sgRNAs (Shan et al. 2013; Ma et al. 2015). Based on homology search by BLAST, we found mRNA sequence of PDS3 from Phalaenopsis equestris orchid (XM_020730635). It is $1767 b p$ from AUG to UAG in length, and total length of mRNA in database is 2176 bp (Figure 2A). The protein motive showed that there is Pf01593 amino oxidase protein family that might be important to function the phytoene desaturase enzyme in carotenoid biosynthesis pathway (Naing et al. 2019). The DNA sequences of $P$. amabilis were selected as the candidates to determine the NGG as the protospacer adjacent motifs (PAM). To study the efficiency of CRISPR/Cas9 genome editing in $P$. amabilis, we selected two targets in PHYTOENE DESATURASE 3 (PDS3) gene. PDS3 is involved in carotenoid synthesis in wich the mutation shown albino phenotype (Qin et al. 2007). Twenty nucleotides were selected from the nucleotide sequence no 312-346 region of the PDS3 genes. Target 1 (PDS3T1) and target 2 (PDS3T2) were separated by $9 \mathrm{bp}$. The sequence of $P D S 3 T 1$ is TTCGTGACTGTTTACGTCCC and PDS3T2 sequence is CTAGAAGTGGTAATTGCTGG (Figure2B).

The specificity of these candidates are examined by BLAST search against the genome sequence of $P$. amabilis. We checked it by BLAST and found P. amabilis PDS3mRNA is expected to be almost identical to that of $P$. equestris based on our sequence data (Figure 2B). In order to make the CRISPR/Cas9 constructs, two DNA oligonucleotides were chemically synthesized for each gRNA. The two oligonucleotides were complementary for the corresponding sequence to the spacer, and the sequences GGC and AAAC were added to the 50 end of the forward and reverse oligonucleotides, respectively, to allow the formation of cohesive ends of BsaI restriction sites following annealing (Kui et al. 2017).

\subsection{Selection of Transformant Protocorms and Plant Regeneration}

After three days of co-cultivation and transformation process have done, the infected protocorms were transferred to selection medium containing $10 \mathrm{ppm}$ hygromisin and incubated for 4 weeks. After that, survived protocorms that indicated transformant candidates with green color were transferred to NP medium with NAA: BA (1:2). Af- 
A
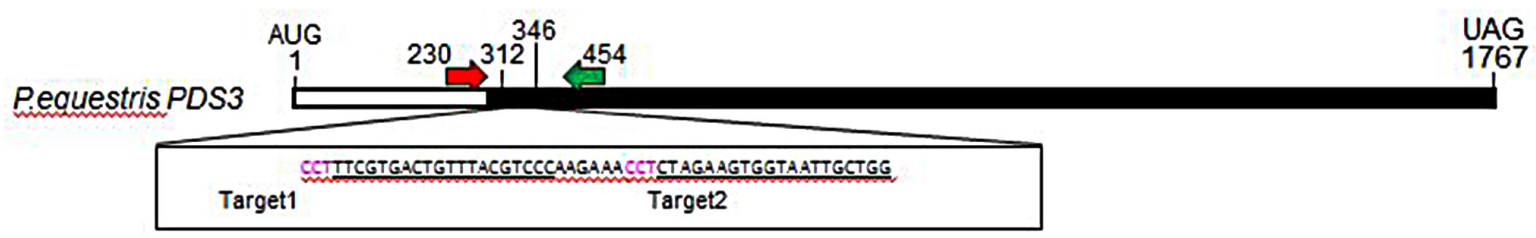

B

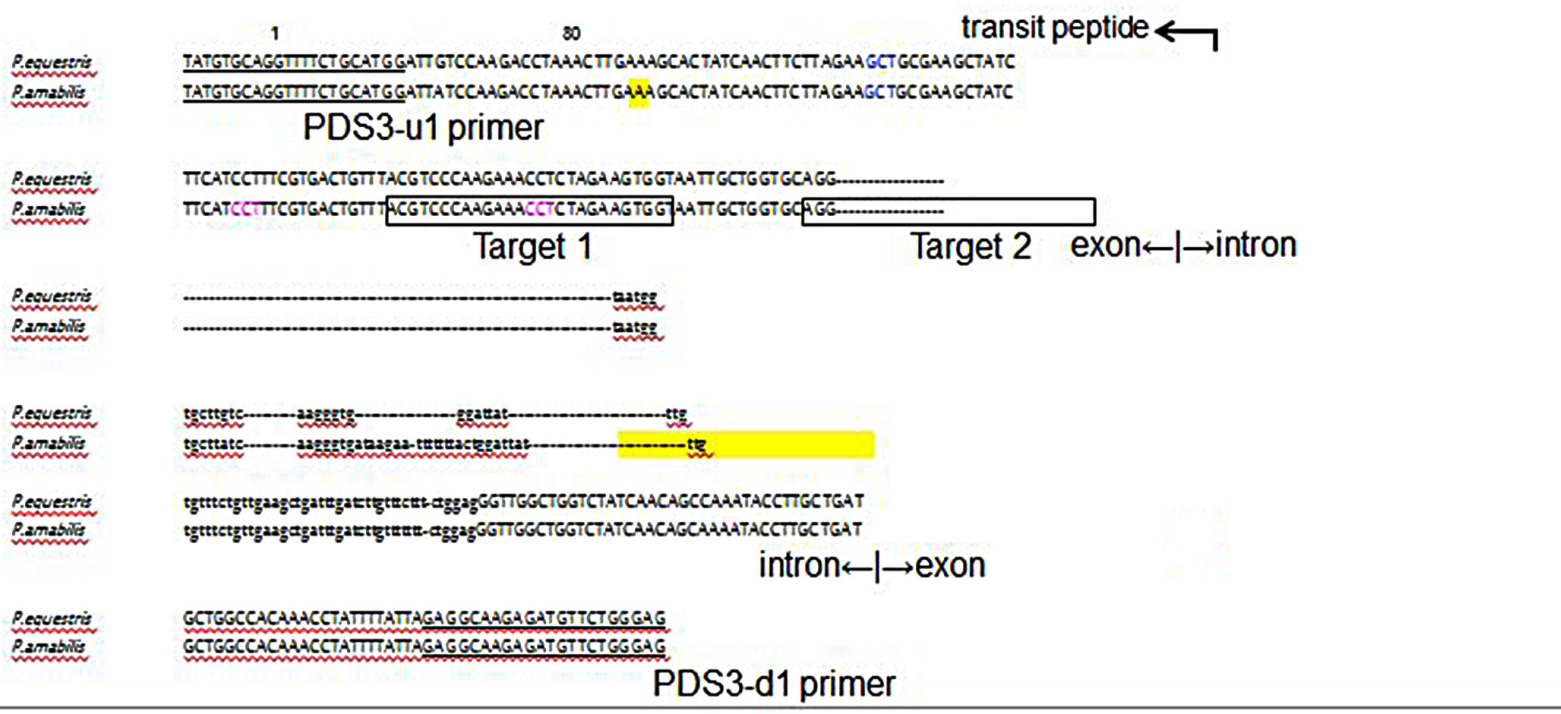

FIGURE 2 Map of PDS3 gene of $P$. equestris and target sequence position for CRISPR/Cas9 Genome editing system. (A) PDS3cDNA of $P$. equestris based on PDS3mRNA sequence from database; (B) PDS3 gene structure allignment of $P$. equestris and $P$. amabilis genom DNA. Red arrow: PDS3-u1 forward primer, green arrow: PDS3-d1 reverse primer, blue box: Predicted protein family region.

ter protocorms grown on the medium, molecular analysis was carried out to see the integration of T-DNA in plant genomes.

TABLE 1 List of primer sequences used to amplify CRISPR target region.

\begin{tabular}{lll}
\hline Primer & Primer Sequences (5' to 3') & $\begin{array}{l}\text { Amplicon } \\
\text { size }\end{array}$ \\
\hline HPT & R: 5' AGGCTATGGATGCGATCGCTG & $545 \mathrm{bp}$ \\
& F: 5' TCGGACGATTGCGTCGCATC & \\
Cas9 & R: 5' TTACTTTTTCTTTTTTGCCTG & $402 \mathrm{bp}$ \\
& F: 5'CACCATGGACTATAAGGACCACGAC \\
PDS & R: 5'CTC CCA GAA CAT CTC TTG CCT C & $280 \mathrm{bp}$ \\
& F: 5' TAT GTG CAG GTT TTC TGC ATG G \\
Trnl-F & R: 5' ATTTGAACTGGTGACACGAG & $1200 \mathrm{bp}$ \\
(Internal & F: 5' CGAAATCGGTAGACGCTACG & \\
control) & & \\
\hline
\end{tabular}

\subsection{Genomic DNA Isolation}

The leaf pieces (200 mg) were collected in a sterile $1.5 \mathrm{~mL}$ microfuge tube. Plant tissues were ground into a fine powder, then added with $500 \mu \mathrm{L}$ of $3 \%$ CTAB buffer. Plant extract mixture were incubated for $30 \mathrm{~min}$ at $60^{\circ} \mathrm{C}$ in water bath. One volume of chloroform:isoamyl alcohol was added, mixed well and incubated for $30 \mathrm{~min}$ in room temperature with shaking for homogenation, then centrifuged the mixture at $5000 \mathrm{rpm}$ for $5 \mathrm{~min}$. The upper phase solution was carefully transferred to a new sterile $1.5 \mathrm{~mL}$ tube. The volume of supernatant was determined. One tenth volume of $\mathrm{Na}$ acetate or cold absolute $\mathrm{EtOH}$ was added and mixed gently, then incubated at $-20^{\circ} \mathrm{C}$ for 10 min. Sample was centrifuged at $1000 \mathrm{rpm}$ for $5 \mathrm{~min}$ and supernatant was discarded. The pellet was washed with $70 \%$ ethanol by centrifugation at $1000 \mathrm{rpm}$ for $2 \mathrm{~min}$. To dry up the DNA pellets, tubes with their lids open were inverted on sterile tissue paper for $30 \mathrm{~min}$ then DNA pellets were dissolved in 25-30 $\mu \mathrm{L}$ TE buffer/ Nuclease free water each. Isolated DNA samples were stored in -20 ${ }^{\circ} \mathrm{C}$. The genomic DNA concentration in resulting solution (200 $\mu \mathrm{L}$ ) was measured by spectrophotometer (Nanodrop 200C, Thermoscientific).

\subsection{PCR test for transformant candidates}

In this study the direct PCR method was performed using KOD Fx-Neo PCR Kit (Toyobo, Japan). The DNA template used was obtained by cutting a small protocorm $( \pm$ $0.05 \mathrm{~cm}$ ) of the plant transformant candidates using sterile tweezers embedded. Samples of plant parts were crushed by mixing $30 \mu \mathrm{L}$ buffer solution (buffer solution A: 100 
$\mu \mathrm{M}$ Tris-HCl pH 9.5; $1 \mathrm{M} \mathrm{KCl;} 10$ mM EDTA) with micropestle. DNA templates were obtained from the supernatant. PCR was performed in a $25 \mu \mathrm{L}$ volume containing $1 \mu \mathrm{L}$ genomic DNA sample, $12.5 \mu \mathrm{L} 2 \times$ PCR buffer for KOD Fx Neo, $5 \mu \mathrm{L} 2 \mathrm{mM}$ dNTPs, $0.5 \mu \mathrm{L}$ KOD Fx Neo (Toyobo, Japan), $0.75 \mu \mathrm{L}$ of each primers and 4.5 $\mu \mathrm{L}$ nuclease-free water. The PCR conditions were predenaturation $\left(94^{\circ} \mathrm{C}\right.$ for $2 \mathrm{~min}$ ), 30 cycles of denaturation (98 ${ }^{\circ} \mathrm{C}$ for $\left.10 \mathrm{~s}\right)$, annealing $\left(58{ }^{\circ} \mathrm{C}\right.$ for $50 \mathrm{~s}$ ), and extension $\left(68^{\circ} \mathrm{C}\right.$ for $\left.45 \mathrm{~s}\right)$. The PCR was performed using T100 TM Thermal Cycler (Bio-Rad). The primer sequences are shown in Table 1.

\subsection{Qualitative analysis of transformant}

To find out whether there is a mutation at the target site in the transformant genome, a PCR with primers specific to the target gene was performed. Plant genome DNA were amplified by PCR using PDS primers and separated the PCR product using 4\% low EEO agarose S (Nippon Gene, 313-90231). The low EEO agarose was dissolved in $1 \times$ Tris-Acetate EDTA (TAE) bufer (40 mM Tris-acetate, 0.5 mM EDTA pH 8.3, Fisher Bioregents BP13324) by heating the solution in a microwave oven for 2-3 min. PCR with some primers: HPT, Cas9 and trnL-F were also conducted using specific primers and checked using 2\% agarose gel (Sigma Aldrich type 2). The melted agarose gels were added with $5 \mu \mathrm{L}$ of ethidium bromide (10 $\mathrm{mg} / \mathrm{mL}$ ), then it was immediately poured on a UV transparent gel casting tray. High concentration of agarose gels should be poured rapidly as the gel solidifies quickly. The electrophoresis chamber filled with $1 \times$ TAE buffer till about $1 \mathrm{~cm}$ above the gel. The total of $10 \mu \mathrm{L}$ of each PCR sample was loaded into each well and electrophoresis was performed for $1 \mathrm{~h}$ and $40 \mathrm{~min}$. Our power supply was set to $100 \mathrm{~V}$. The $4 \%$ agarose gels were run at room temperature $\left(25^{\circ} \mathrm{C}\right)$. DNA size marker $1 \mathrm{~kb}$ (Geneaid) is a readyto-use solution containing $6 \times$ loading dyes. Gel images were acquired using a regular gel-documentation system (Bhattacharya and Van Meir 2019).

\section{Results and Discussion}

\subsection{Plant genetic transformation mediated by Agrobacterium tumefaciens}

In this research protocorms were used for transformation material using Agrobacterium tumefaciens strain EHA105 carried T-DNA with UBI::Cas9::U3::PDS in pRGEB32. The transformation efficiency in $P$. amabilis using PDS3T1 sgRNA is $0.9 \%$, lower than transformation efficiency using PDS3T2 sgRNA is $0.96 \%$ (Table 2). Transformation efficiency in this study were lower compared to the previous study. In Dendrobium phalaenopsis, transformation efficiency reached until 12\% (Setiari et al. 2018) and D. lasianthera up to 70\% (Utami et al. 2018). This might be caused by each species has their own genetic make up (Men et al. 2003). Moreover, transformation by using Agrobacterium was a biological approach that has various factor to get an optimum result, such as type explant, bacteria condition and pre-treatment (Chen et al. 2018).

Agrobacterium-mediated transformation is a method that enables production of stable transformants and as a model for studying the cellular localization and interactions between proteins that are important for the development of functional genomic analysis (Krenek et al. 2015). Agrobacterium-mediated transformation used by scientists can introduce new traits into plants through overexpression, knockdown or knockout the gene expression through Agrobacterium-mediated T-DNA transfer (Semiarti et al. 2007; Guo et al. 2019). A. tumefaciens uses a unique virulence mechanism to induce tumors, it delivers DNA fragments (transferred DNA or T-DNA) to host cells where the T-DNA ultimately integrates into the genome. The pathogenicity of A. tumefaciens is predominantly determined by a large $\mathrm{Ti}$ (tumor inducing) plasmid (Guo et al. 2019).

The phenotypic change was also detected from the green color reduction of almost all protocorms, that changes from green to black on the 4th week in the selection medium. The black phenotype of protocorm can be

TABLE 2 Efficiency of transformation in Protocorm P. amabilis.

\begin{tabular}{|c|c|c|c|c|c|c|c|c|}
\hline \multirow{2}{*}{ Treatment } & \multirow{2}{*}{ Repetition } & \multirow{2}{*}{$\begin{array}{l}\Sigma \text { transformed } \\
\text { protocorm }\end{array}$} & \multirow{2}{*}{$\begin{array}{c}\sum \text { survival protocorms } \\
\text { in selection medium }\end{array}$} & \multicolumn{3}{|c|}{$\begin{array}{c}\text { PCR } \\
\text { Detection }\end{array}$} & \multirow[t]{2}{*}{$\begin{array}{c}\text { Efficiency of } \\
\text { transformation (\%) }\end{array}$} & \multirow[t]{2}{*}{$\begin{array}{c}\text { Efficiency of } \\
\text { Genome editing (\%) }\end{array}$} \\
\hline & & & & HPT+ & Cas9+ & PDS+ & & \\
\hline Non-transformant & 1 & 430 & 3 & $0 / 3$ & $0 / 3$ & $0 / 3$ & 0 & \\
\hline \multirow{3}{*}{ (Wild Type) } & 2 & 405 & 2 & $0 / 2$ & $0 / 2$ & $0 / 2$ & 0 & \\
\hline & 3 & 392 & 1 & $0 / 1$ & $0 / 1$ & $0 / 1$ & 0 & \\
\hline & Total & 1227 & 6 & $0 / 6$ & $0 / 6$ & $0 / 6$ & 0 & 0 \\
\hline \multirow[t]{4}{*}{ PDS3T1 } & 1 & 420 & 4 & $1 / 7$ & $1 / 7$ & $1 / 7$ & 0.95 & \\
\hline & 2 & 385 & 3 & $1 / 3$ & $1 / 3$ & $1 / 3$ & 0.77 & \\
\hline & 3 & 295 & 3 & $1 / 4$ & $1 / 4$ & $1 / 4$ & 1.0 & \\
\hline & Total & 1100 & 10 & $3 / 14$ & $3 / 14$ & $3 / 14$ & 0.90 & 0.19 \\
\hline \multirow[t]{4}{*}{ PDS3T2 } & 1 & 417 & 5 & $1 / 5$ & $1 / 5$ & $1 / 5$ & 1.1 & \\
\hline & 2 & 407 & 4 & $1 / 4$ & $1 / 4$ & $1 / 4$ & 0.98 & \\
\hline & 3 & 365 & 3 & $1 / 3$ & $1 / 3$ & $1 / 3$ & 0.82 & \\
\hline & Total & 1189 & 12 & $3 / 12$ & $3 / 12$ & $3 / 12$ & 0.96 & 0.25 \\
\hline
\end{tabular}


caused by hygromycin as a selection agent affected photosynthesis rate. As Mingeot-Leclercq et al. (1999), this antibiotic can bind 80 ribosomal complexes. Furthermore, the green protocorm was indicated as a transformant candidate plant (Figure 3).

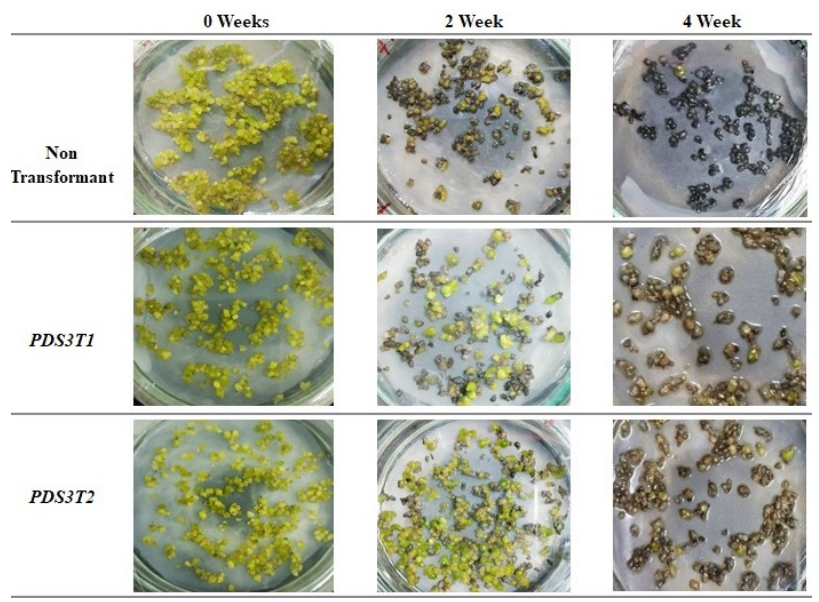

FIGURE 3 Development of $P$. amabilis protocorms on Hygromycin containing selection medium for transformants.

In this result the transformation efficiency was low (Table 2), it might be due to the low efficiency in TDNA integration into the plant genome. Our previous study in Dendrobium phalaenopsis, efficiency transformation reached until 12\% (Setiari et al. 2018) and Utami et al. (2018) got high transformation efficiency in $D$. lasianthera that the transformation efficiency up to $70 \%$. This might be caused by specific character of each species related to their own genetic make up (Men et al. 2003). Moreover, transformation by using Agrobacterium was a biological approach that has various factor to get an optimum result, such as type of explant, bacteria condition and pre-treatment (Chen et al. 2018). A. tumefaciensmediated genetic transformation has been routinely used in rice. The study used different cultivar rice with $A$. tumefaciens-mediated genetic transformation. However, the transformation efficiency of the indica rice variety is still much lower than that of japonica cultivars. This further improvement on the transformation efficiency lies in the genetic manipulation of the plant itself, which requires a better understanding of the underlying process in account for the susceptibility of plant cells to Agrobacterium infection as well as the identification of plant genes involved in the transformation process (Tie et al. 2012). Utami et al. (2018) used the five different infection period of Agrobacterium for another purpose. According to our result, futher research is necessary to increase mutation frequency for mutant propagation.

\subsection{PCR-based detection of CRISPR/Cas9-edited in transformant plant}

Transformant candidates were screened using PCR to confirm T-DNA integration. HPT and Cas9 primers were used to screen the candidates to confirm the transformations of pRGEB32 construct harboring T-DNA (UBI :: Cas9 :: U3
:: PDS). After that, 8 weeks-old green protocorms grown on selection medium were used as screening materials. We regenerated the transformant in NP media+ ZPT (NAA: BA 1:2). Protocorms were grown in regeneration media until they produced leaves for mutation detection according to Bhattacharya and Van Meir (2019) method.

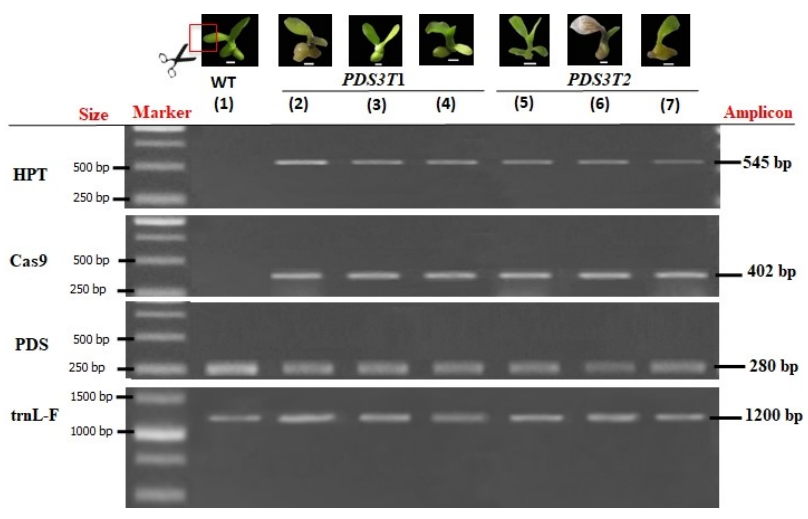

FIGURE 4 Detection of transformant orchid plants based on PCRamplicon of transgene. PCR were performed using specific primers of HPT, Cas9, and PDS3 genes. The fragment of trnL-F was used as internal control of PCR reaction. (M: $1 \mathrm{~kb}$ Gene Aid DNA Marker, 1 Wild type P.amabilis, 2-4: Transformant candidates PDS3T1, and 5-7: Transformant candidates PDS3T2).

The electrophoresis results (Figure 4) show that TDNA has successfully entered the plant genome. This refers to the band that is detected appearing on some of the primers used. Results showed specific size of amplicon from each primer pairs, i.e HPT (504 bp), Cas9 (402 bp), PDS (280 bp) and trnL-F (1200 bp) as an internal control. Results showed no multiband or band shift on gel electrophoresis. This possibility does not occur in the genome insertion so that no extra thickening of the band or bands appear. So it needs to be confirmed by using Sanger Sequencing analysis method to see whether there are the mutations occurred or not.

\subsection{Sequence analysis of CRISPR/Cas9-edited in transformant plant}

Mutation detection in the genomes of transformant plants were analysed by using 4\% agarose gel low EEO following the protocol of Bhattacharya and Van Meir (2019). Nevertheless, the drawback is that the amplicon size cannot be known accurately. It can only be estimated its size by comparing non-transformant with transformants and referring to the markers used. Further confirmation is required by sequencing and alignment to find specific changes in target sequence. In this research, we compared sequencing data from wild type/non transformant and transformant. We used PDS3 gene with two target site namely PDS3T1 and PDS3T2.

Sequencing analysis uses PCR amplicons produced from genomic DNA templates. Furthermore, the process of alignment of the transformant genomic DNA template that is identical to wild type sequences. This analysis showed that one of the edited protocorms kept a mutation (Figure 5) located on the CRISPR/Cas9 target site for 


\section{PAM \\ Pa_Wild Type ACTGTTTACGTCCCAAGAAAC Pa_PDS3T2 (T-6) ACTGTTTACGTCCCAAGAAACCTCTAGAAGTGGTAAT-G-TGG' Target Site CTAGAAGTGGTAATTGCTGG}

FIGURE 5 The sequence alignment of the edited transformant PaPDS3T2 line against wild-type sequence produced in protocorm of P.amabilis. Highlighted nucleotide by red there indication has occurred mutation.

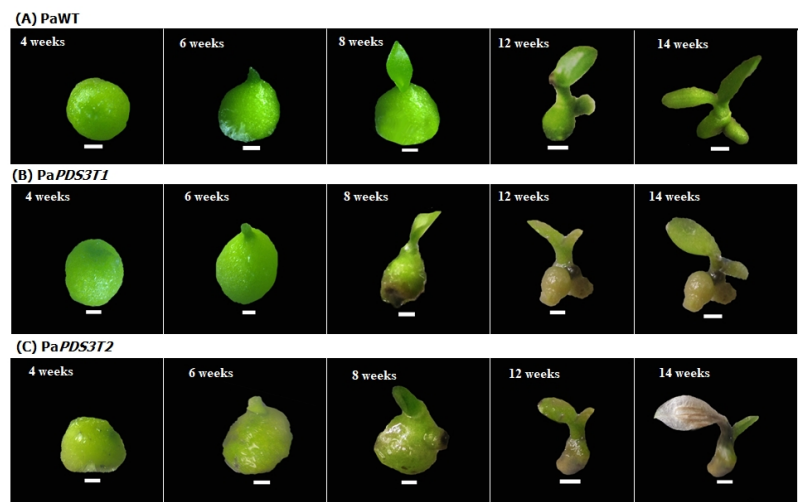

FIGURE 6 Phenotypic changes on the development of protocorm into shoot of $P$. amabilis. (A) Wild-type and the transformant (B) PDS3T1; (C) PDS3T2.

PDS3T2. This indicated that mutations occured as a base deletions due to internal DNA repair, then the process of cutting double strand DNA and forming a double-strand break were caused by CRISPR/Cas9. The repair process is called NHEJ (Non-homologous end joining) gene mutation that results in a knock-out. This mutation induces changes in amino acid sequences which will also changes the phenotypic. Because of those carotenoid pathways disrupted causing an albino phenotype (Figure 6). In our previous study, to edit the homologous PDS3 gene in P. amabilis orchids, we used the pKIR1.1 vector (Tsutsui and Higashiyama 2016) with the AtRPS5A promoter from Arabidopsis, but we did not get any mutants that showed insertion or deletion at the target site of PDS3 gene. The pRGEB32 vector that containing OsUbi-pro, a Ubiquitin promoter from rice (Oryza sativa) which is a monocot plant similar to an orchid, turns out that CRISPR/Cas9 can work properly in orchid, produced orchid mutant with color changes on the leaves because of the indel occurances in their $P D S 3$ target sites.

Our in silico sequence allignment of the wild type and CRISPR/Cas9-edited P. amabilis T\#6 PDS3T2 (Figure 7) showed that deletion changed the translated amino acid sequence. Indicated by black triangles. Yellow highlight was PAM sequence. This data indicated that CRISPR/Cas9 can induced a mutation in the way of deletion. This phenomenon lead a frameshift on the translated amino acid sequence.

This shows that the CRISPR/Cas9 method can be used to edit orchid genome using appropriate vectors and pro-

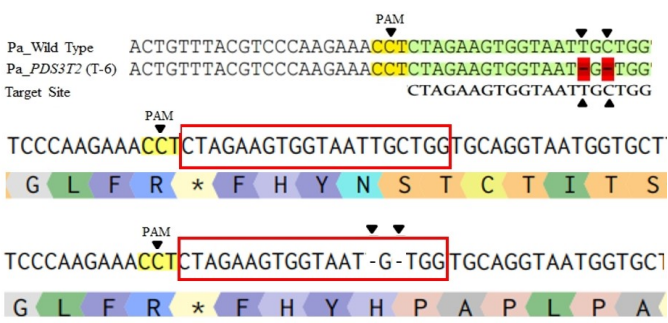

FIGURE 7 In silico Sequence alignment of Wildtype and edited $P$. amabilis T\#6 PDS3T2 in Benchling. Highlighted nucleotide by black triangles showed where the change made by CRISPR/Cas9 and changing the translated amino acid sequence.

moters.

\section{Conclusions}

CRISPR/Cas9 genome editing KO system can be applied to orchids, especially Phalaenopsis amabilis. The PHYTOENE DESATURASE 3 (PDS3) gene can be used as a model/marker gene which makes it easy to determine whether the two PDS3 alleles are disturbed in the observed tissue. The pds3 mutants of $P$. amabilis show albino phenotype in the leaf tissues. The use of CRISPR/Cas9 for $P$. amabilis is still in its early stages in the T0 generation, so further research is needed for the the next generation in order to obtain precise information about the effectiveness next generation of the editing genome in orchids, especially for $\mathrm{KO}$ suppressor functional genes in producing new trait.

\section{Acknowledgments}

This work was supported by Basic Research Grant from the Indonesian Ministry of Research, Technology and Higher Education with Contract No. 2791/UN1/DITLIT/DIT-LIT/LT/2019 given to E.S. as PI (2018-2019), and in part was supported by Japan Society for Promotion of Science (JSPS) Fiscal Year 2018-2019 with Contract No:219/D3/PG/2019 given to E.S. as PI.

\section{Authors' contributions}

ES, AP, JW, SM and YY designed the research. YY, KN and YA designed the sgRNA in plasmid pRGEB32 cas- 
settes. SN, YS and MDL conducted the genetic transformation bacteria and screening in antibiotic supplemented plates. SN conducted genetic transformation and YS conducted the isolation of genomic DNA, PCR. SN conducted, sequencing, subsequent analyzes, data compiling and wrote the manuscript. All authors read and approved the final version of the manuscript.

\section{Competing interests}

The authors declare no competing interest.

\section{References}

Bhattacharya D, Van Meir EG. 2019. A simple genotyping method to detect small CRISPR-Cas9 induced indels by agarose gel electrophoresis. Sci Rep. 9(4437). doi:10.1038/s41598-019-39950-4.

Cai J, Liu X, Vanneste K, Proost S, Tsai WC, Liu KW, Chen LJ, He Y, Xu Q, Bian C, et al. 2015. The genome sequence of the orchid Phalaenopsis equestris. Nat Genet. 47(1):65-72. doi:10.1038/ng.3149.

Chen J, Wang L, Chen J, Huang J, Liu F, Guo R, Yang L, Grabon A, Zhao K, Kong F, Others. 2018. Agrobacterium tumefaciens-mediated transformation system for the important medicinal plant Dendrobium catenatum Lindl. In Vitro Cell Dev Biol Plant. 54(3):228239. doi:10.1007/s11627-018-9903-4.

Chiruvella KK, Liang Z, Wilson TE. 2013. Repair of Double-Strand Breaks by End Joining. Cold Spring Harbor Perspect Biol. 5(5):a012757. doi:10.1101/cshperspect.a012757.

Guo M, Ye J, Gao D, Xu N, Yang J. 2019. Agrobacterium-mediated horizontal gene transfer: Mechanism, biotechnological application, potential risk and forestalling strategy. Biotechnol Adv. 37(1):259-270. doi:10.1016/j.biotechadv.2018.12.008.

Hahn F, Eisenhut M, Mantegazza O, Weber APM. 2018. Homology-Directed Repair of a Defective Glabrous Gene in Arabidopsis With Cas9-Based Gene Targeting . doi:10.3389/fpls.2018.00424.

Hsu PD, Lander ES, Zhang F. 2014. Development and Applications of CRISPR-Cas9 for Genome Engineering. Cell. 157(6):1262-1278. doi:10.1016/j.cell.2014.05.010.

Koschmieder J, Fehling-Kaschek M, Schaub P, Ghisla S, Brausemann A, Timmer J, Beyer P. 2017. Plant-type phytoene desaturase: Functional evaluation of structural implications. PLoS ONE. 12(11):e0187628. doi:10.1371/journal.pone.0187628.

Krenek P, Samajova O, Luptovciak I, Doskocilova A, Komis G, Samaj J. 2015. Transient plant transformation mediated by Agrobacterium tumefaciens: Principles, methods and applications. Biotechnol Adv. 33(6, Part 2):1024-1042. doi:10.1016/j.biotechadv.2015.03.012.

Kui L, Chen H, Zhang W, He S, Xiong Z, Zhang
Y, Yan L, Zhong C, He F, Chen J, et al. 2017. Building a Genetic Manipulation Tool Box for Orchid Biology: Identification of Constitutive Promoters and Application of CRISPR/Cas9 in the Orchid, Dendrobium officinale. Front Plant Sci. 7:2036. doi:10.3389/fpls.2016.02036.

Liu X, Wu S, Xu J, Sui C, Wei J. 2017. Application of CRISPR/Cas9 in plant biology. Acta Pharm Sin B. 7(3):292-302. doi:10.1016/j.apsb.2017.01.002.

Ma X, Zhang Q, Zhu Q, Liu W, Chen Y, Qiu R, Wang B, Yang Z, Li H, Lin Y, et al. 2015. A Robust CRISPR/Cas9 System for Convenient, HighEfficiency Multiplex Genome Editing in Monocot and Dicot Plants. Mol Plant. 8(8):1274-1284. doi:10.1016/j.molp.2015.04.007.

Men S, Ming X, Liu R, Wei C, Li Y. 2003. Agrobacterium-mediated genetic transformation of a Dendrobium orchid. Plant Cell, Tissue Organ Cult. 75(1):6371. doi:10.1023/A:1024627917470.

Mingeot-Leclercq MP, Glupczynski Y, Tulkens PM. 1999. Aminoglycosides: activity and resistance. Antimicrob Agents Chemother. 43(4):727-737.

Naing AH, Kyu SY, Pe PPW, Park KI, Lee JM, Lim KB, Kim CK. 2019. Silencing of the phytoene desaturase (PDS) gene affects the expression of fruitripening genes in tomatoes. Plant Methods 15(1):110. doi:10.1186/s13007-019-0491-z.

Qin G, Gu H, Ma L, Peng Y, Deng XW, Chen Z, Qu LJ. 2007. Disruption of phytoene desaturase gene results in albino and dwarf phenotypes in Arabidopsis by impairing chlorophyll, carotenoid, and gibberellin biosynthesis. Cell Res. 17(5):471-482. doi:10.1038/cr.2007.40.

Semiarti E, Indrianto A, Purwantoro A, Isminingsih S, Suseno N, Ishikawa T, Yoshioka Y, Machida Y, Machida C. 2007. Agrobacterium-mediated transformation of the wild orchid species Phalaenopsis amabilis. Plant Biotechnol. 24(3):265-272. doi:10.5511/plantbiotechnology.24.265.

Semiarti E, Indrianto A, Purwantoro A, Machida Y, Machida C. 2011. Agrobacterium-Mediated Transformation of Indonesian Orchids for Micropropagation. In: M Alvarez, editor, Genetic Transformation. Rijeka: IntechOpen. p. Ch. 11. doi:10.5772/24997.

Semiarti E, Indrianto A, Purwantoro YH, Martiwi INA, Feroniasanti YML, Nadifah F, Mercuriana IS, Dwiyani R, Iwakawa H, Yoshioka Y, et al. 2010. High-frequency genetic transformation of Phalaenopsis amabilis orchid using tomato extractenriched medium for the pre-culture of protocorms. J Hortic Sci Biotechnol. 85(3):205-210. doi:10.1080/14620316.2010.11512655.

Setiari N, Purwantoro A, Moeljopawiro S, Semiarti E. 2018. Micropropagation of Dendrobium phalaenopsis Orchid Through Overexpression of Embryo Gene AtRKD4. AGRIVITA, Journal of Agricultural Science 40(2):284-294. doi:10.17503/agrivita.v40i2.1690. 
Shan Q, Wang Y, Li J, Zhang Y, Chen K, Liang Z, Zhang K, Liu J, Xi JJ, Qiu JL, Gao C. 2013. Targeted genome modification of crop plants using a CRISPR-Cas system. Nat Biotechnol. 31(8):686688. doi:10.1038/nbt.2650.

Shen H, Strunks GD, Klemann BJPM, Hooykaas PJJ, de Pater S. 2017. CRISPR/Cas9-Induced DoubleStrand Break Repair in Arabidopsis Nonhomologous End-Joining Mutants. G3: Genes, Genomes, Genet. 7(1):193-202. doi:10.1534/g3.116.035204.

Tie W, Zhou F, Wang L, Xie W, Chen H, Li X, Lin Y. 2012. Reasons for lower transformation efficiency in indica rice using Agrobacterium tumefaciens-mediated transformation: lessons from transformation assays and genome-wide expression profiling. Plant molecular biology 78(1-2):1-18. doi:10.1007/s11103-011-9842-5.

Tong CG, Wu FH, Yuan YH, Chen YR, Lin CS. 2019. High-efficiency CRISPR/Cas-based editing of Phalaenopsis orchid MADS genes. Plant Biotechnol J. 18(4):889-891. doi:10.1111/pbi.13264.

Tsutsui H, Higashiyama T. 2016. pKAMA-ITACHI Vectors for Highly Efficient CRISPR/Cas9-Mediated Gene Knockout in Arabidopsis thaliana. Plant Cell Physiol. 58(1):46-56. doi:10.1093/pcp/pcw191.

Utami ESW, Hariyanto S, Manuhara YSW. 2018. Agrobacterium tumefaciens-mediated transformation of Dendrobium lasianthera JJ Sm: An important medicinal orchid. J Genet Eng Biotechnol. 16(2):703-709. doi:10.1016/j.jgeb.2018.02.002.

Wang M, Wang G, Ji J, Wang J. 2009. The effect of pds gene silencing on chloroplast pigment composition, thylakoid membrane structure and photosynthesis efficiency in tobacco plants. Plant Sci. 177(3):222-226. doi:10.1016/j.plantsci.2009.04.006.

Xie K, Minkenberg B, Yang Y. $2015 . \quad$ Boosting CRISPR/Cas9 multiplex editing capability with the endogenous tRNA-processing system. Proc Natl Acad Sci. 112(11):3570-3575. doi:10.1073/pnas.1420294112. 